\title{
Free $\alpha$-Globin Pool in Human Bone Marrow
}

\author{
Frances M. Gill and Elias Schwartz \\ From the Children's Hospital of Philadelphia, and the Department of Pediatrics, \\ University of Pennsylvania School of Medicine, Philadelphia, Pennsylvania 19146
}

\begin{abstract}
A в S T R A C T A pool of free $\alpha$-globin chains was found in the bone marrow samples from three controls, two patients with $\beta$-thalassemia trait, three with sickle $\beta$-thalassemia, three with hemoglobin $(\mathrm{Hb})$ Lepore trait, one with $\alpha \beta$-thalassemia, four with homozygous $\beta$-thalassemia, and one doubly heterozygous for $\mathrm{Hb}$ Lepore and $\beta$-thalassemia. The average percentage of newly synthesized $\alpha$-chains found in the free $\alpha$-globin pool was $6.2 \%$ in the controls and $33.0 \%$ in the patients heterozygous for thalassemia or $\mathrm{Hb}$ Lepore. These controls and patients had balanced $\beta$ - and $\alpha$-globin synthesis in the bone marrow. In the homozygous patients and in the one patient doubly heterozygous for thalassemia and $\mathrm{Hb} \mathrm{Le}-$ pore, there was a marked deficit of $\beta$-chain synthesis in the bone marrow and also a large pool of newly synthesized free $\alpha$-chains. The function of this pool of free $\alpha$-chains is not known, but it may be involved in the regulation of globin chain synthesis in normal patients and in the compensatory synthesis of $\beta$-chains that occurs in the bone marrow of patients heterozygous for thalassemia or for $\mathrm{Hb}$ Lepore.
\end{abstract}

\section{INTRODUCTION}

In normal persons and in nonthalassemic patients with hemolytic anemia, globin synthesis studies have demonstrated that the production of $\alpha$-chain of normal adult hemoglobin ( $\mathrm{Hb} \mathrm{A}$ ) is equal to that of $\beta$-chain in the peripheral blood reticulocytes (3-5). A very small pool of free $\alpha$-chains has been demonstrated in peripheral blood of nonthalassemic patients by a sensitive method using gel filtration chromatography $(6,7)$. In patients with homozygous $\beta$-thalassemia there is a marked decrease in synthesis of $\beta$-chains $(3,4,8)$. Gel filtration studies have shown a large pool of free $\alpha$-chains in

Preliminary reports have appeared in abstract form $(1,2)$.

Dr. Gill was the recipient of a National Institutes of Health Special Fellowship (HD 54150).

Received for publication 8 May 1973 and in revised form 2 July 1973.

The Journal of Clinical Investigation 、Volume 52 December 1973·3057-306.3 peripheral blood and bone marrow from these patients $(6,7,9)$. In patients with heterozygous $\beta$-thalassemia there is decreased $\beta$-globin production in the peripheral blood reticulocytes $(4,10-12)$. As expected, these patients have a pool of radioactive free $\alpha$-chains in the peripheral blood larger than that found in control patients $(6,7,9,13)$. We have previously described balanced globin chain synthesis in the bone marrow of patients with heterozygous $\beta$-thalassemia and related disorders (14-16). The gel filtration studies in this paper demonstrate the existence of a small pool of free $\alpha$-chains in normal human bone marrow and a much larger pool in patients with heterozygous $\beta$-thalassemia, sickle-thalassemia, and $\mathrm{Hb}$ Lepore trait. These findings are contrary to expectation since these patients had balanced globin synthesis in the bone marrow.

\section{METHODS}

Patients. The patients studied, several of whom have been described previously, included one Italian and one Iraqi with $\beta$-thalassemia trait (16), two American Negroes and one Italian with sickle $\beta$-thalassemia (16), three Italians with $\mathrm{Hb}$ Lepore trait (15), one Italian man doubly heterozygous for $\alpha$ - and $\beta$-thalassemia ( $\alpha \beta$-thalassemia), four Italian children with homozygous $\beta$-thalassemia, and one Italian child doubly heterozygous for $\mathrm{Hb}$ Lepore and high $\mathrm{Hb} \mathrm{A}_{2} \beta$-thalassemia. The patients with $\beta$-thalassemia trait had increased levels of $\mathrm{Hb} \mathrm{A}$, hypochromia, microcytosis, and normal serum iron levels. The patients with sickle $\beta$-thalassemia each had predominantly $\mathrm{Hb} \mathrm{S}, 7-15 \% \mathrm{Hb} \mathrm{A}$, and red cell morphology consistent with the disorder. The patient with $\alpha \beta$-thalassemia had a $\mathrm{Hb} \mathrm{A}_{2}$ of $4.5 \%$, a son with $\beta$-thalassemia major, a peripheral blood $\beta / \alpha$ ratio of 0.90 , and a father and a brother with $\alpha$-thalassemia trait. Three children with homozygous $\beta$-thalassemia and one with $\mathrm{Hb}$ Lepore $\beta$-thalassemia were transfused every 2-4 wk. A 2-yr-old child with homozygous $\beta$-thalassemia (A. C.) had not been previously transfused. Three nonthalassemic control subjects with reticulocytosis were also studied.

Measurements of globin chain synthesis. Globin chain synthesis was studied by methods previously described (1618). Peripheral blood or bone marrow was incubated for 2 $\mathrm{h}$ at $37^{\circ} \mathrm{C}$ with $\left[{ }^{14} \mathrm{C}\right]$ leucine, hemolysate was prepared, and the cells were washed three times with isotonic saline. 
TABLE I

Subunit Synthesis and Free $\alpha$-Chain Pool Data

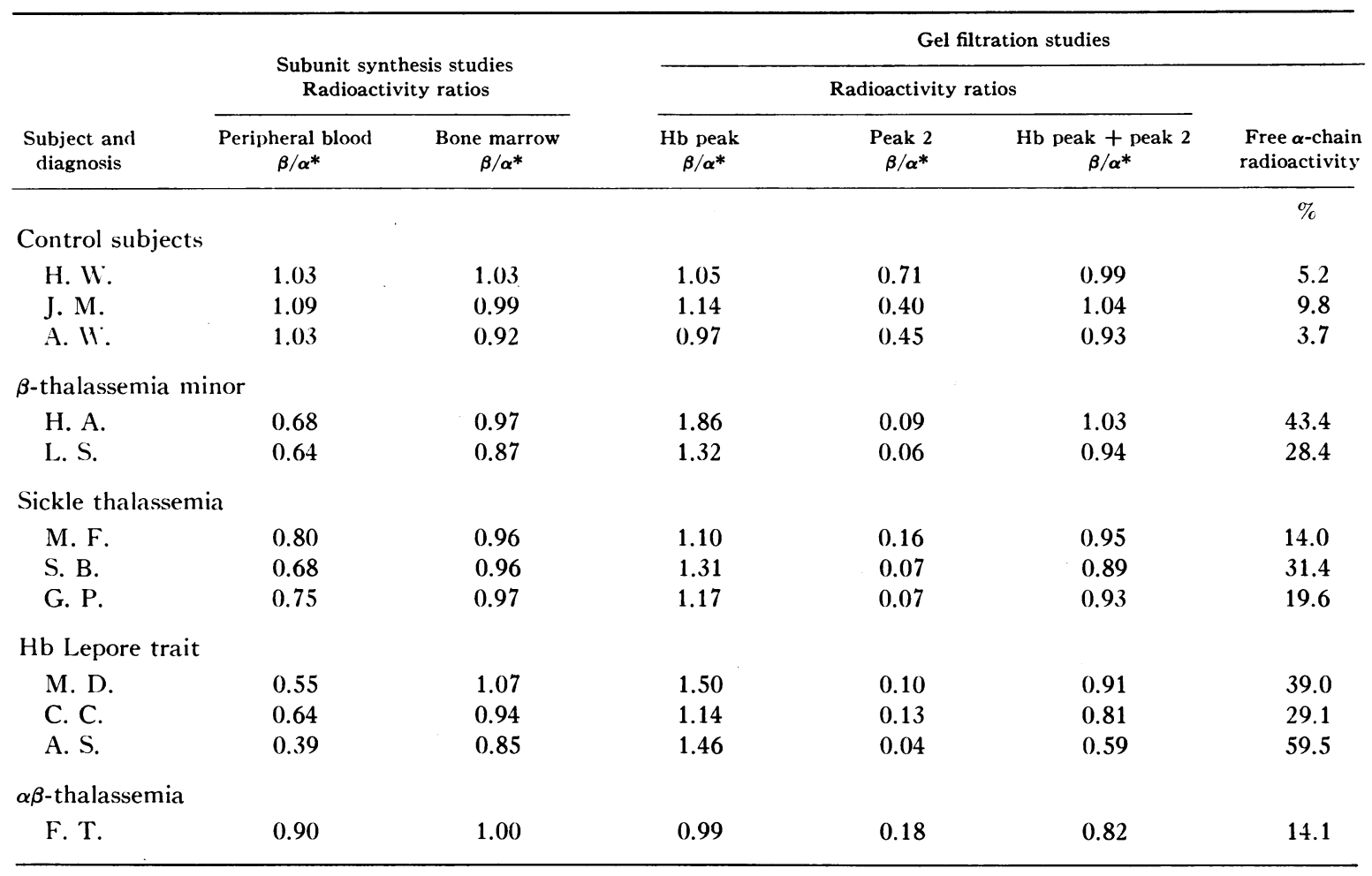

${ }^{*} \beta / \alpha$ ratio refers to $\beta^{\mathrm{A}} / \alpha,\left(\beta^{\mathrm{A}}+\beta^{\mathrm{S}}\right) / \alpha$, or $\left(\beta^{\mathrm{A}}+\right.$ Lepore $) / \alpha$ where appropriate.

The red cells were lysed with 4 vol of $0.001 \mathrm{M} \mathrm{MgCl}$, and isotonicity was restored by adding 1 vol of $1.5 \mathrm{M} \mathrm{KCl}$ Membranes were removed by centrifugation at $12,000 \mathrm{rpm}$ for $45 \mathrm{~min}$. The globin chains were separated by column chromatography. The total radioactivity and specific activity of each chain were determined.

Studies of free $\alpha$-chain pool. 1-ml samples of bone marrow hemolysates were applied immediately after preparation to columns containing Sephadex G-100. Two gel filtration columns connected in series, each $75 \times 0.9 \mathrm{~cm}$, were used for each experiment. The separations took place at $4^{\circ} \mathrm{C}$ with a $0.1 \mathrm{M}$ phosphate buffer at $\mathrm{pH} 7.0$. 2-ml fractions were collected with an elution rate of $3 \mathrm{ml} / \mathrm{h}$. Radioactivity of a $0.2-\mathrm{ml}$ sample from each tube was measured in a liquid scintillation counter. The degree of quenching of each sample was determined by the subsequent addition of an internal standard $\left(\left[{ }^{14} \mathrm{C}\right]\right.$ toluene $) .5 \mathrm{ml}$ of barbital buffer $(\mathrm{pH} 8.6,0.05 \mathrm{M})$ was then added to each tube, and optical density at $540 \mathrm{~nm}$ of each fraction was measured. The fractions contained in the major peak of radioactivity that coincided with the hemoglobin peak of optical density at $540 \mathrm{~nm}$ were pooled ( $\mathrm{Hb}$ peak). In control subjects a small shoulder of radioactivity eluted after the hemoglobin peak. The tubes comprising the shoulder were pooled (peak 2). In patients with $\beta$-thalassemia or $\mathrm{Hb}$ Lepore one or two distinct radioactivity peaks eluted after the hemoglobin peak. The tubes in each of these peaks were pooled separately (peak 2 , peak 3 ). $40 \mathrm{mg}$ of nonradioactive hemoglobin from a normal adult were added to the $\mathrm{Hb}$ peak, and $80 \mathrm{mg}$ were added to peak 2 and to peak 3 as carrier protein.

Globin was prepared from each peak (18), and the globin chains were separated by column chromatography on carboxymethyl cellulose at $\mathrm{pH} 6.7$ in $8 \mathrm{M}$ urea (17, 18). For each of the collected fractions the optical density at $280 \mathrm{~nm}$ and the radioactivity of a $1.0-\mathrm{ml}$ sample were measured. The total radioactivity of each globin chain was determined by adding the separate radioactivities of the fractions contained in that peak. The relative size of the free $\alpha$-chain pool was calculated by adding the total radioactivities of $\alpha$-chain in peaks 2 and 3, subtracting a correction for $\alpha$-chain attached to $\beta$-chain present in peaks 2 and 3 , and dividing the difference by the total radioactivity of $\alpha$-chain in the $\mathrm{Hb}$ peak, peak 2, and peak 3 . The correction for $\alpha$-chain combined with radioactive $\beta$ chain in peaks 2 and 3 was obtained by dividing the total radioactivity in $\beta$-chain in those peaks by the $\beta / \alpha$ ratio of radioactivities determined from the $\mathrm{Hb}$ peak.

\section{RESULTS}

Table I summarizes the results of globin synthesis studies of peripheral blood and bone marrow. The five patients with $\beta$-thalassemia trait or sickle $\beta$-thalassemia and the three with $\mathrm{Hb}$ Lepore trait each had a decreased $\beta / \alpha$ ratio in the peripheral blood and a ratio close to one in the bone marrow. The mean of the peripheral blood 
$\beta / \alpha$ ratios was $0.64 \pm 0.12(1 \mathrm{SD})$ while the mean of the bone marrow ratios was $0.95 \pm 0.06$. The peripheral blood ratios are similar to those found in Italian patients with $\beta$-thalassemia trait by us $(0.57 \pm 0.08)$ and by others $(4,10-12)$. The patient with $\alpha \beta$-thalassemia had normal $\beta / \alpha$ ratios in peripheral blood and bone marrow $(0.90$, $1.00)$. The three controls had peripheral blood ratios from 0.97 to 1.05 and bone marrow ratios from 0.99 to 1.09. Previous controls studied in our laboratory had a mean peripheral blood $\beta / \alpha$ ratio of $0.99 \pm 0.05$. Four previous control bone marrows had $\beta / \alpha$ ratios from 0.94 to 1.06 .

Fig. 1 shows representative elution patterns by gel filtration chromatography for a control subject and patients with heterozygous and homozygous $\beta$-thalassemia. A shoulder of radioactivity is present on the downward slope of the hemoglobin peak from the control. A larger, distinct second peak is present in the sample from the patient with $\beta$-thalassemia trait, while two peaks of molecular weight less that that of hemoglobin are present in the sample from the patient with homozygous

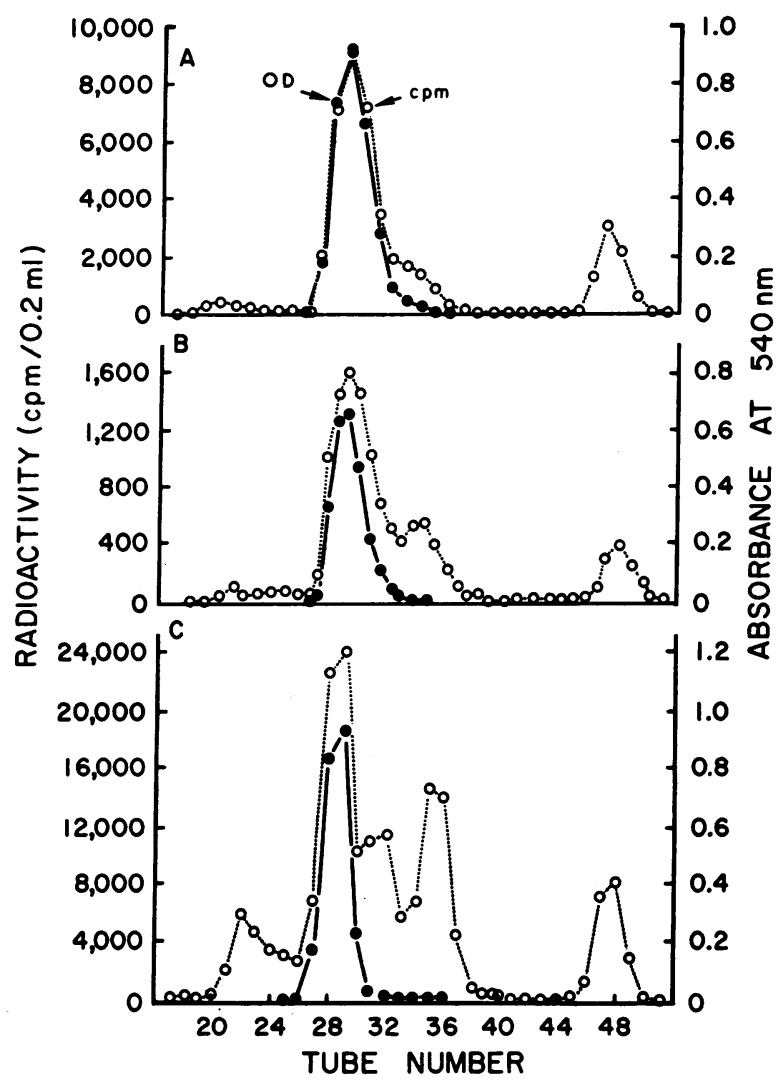

Figure 1 Gel filtration elution patterns from bone marrows of three patients. A. Normal (H. W.) ; B. $\beta$-thalassemia minor (H. A.) ; C. $\beta$-thalassemia (L. C.). The initial peak at tubes $20-24$ contains the void volume, and the leucine elutes at tubes $46-50$.
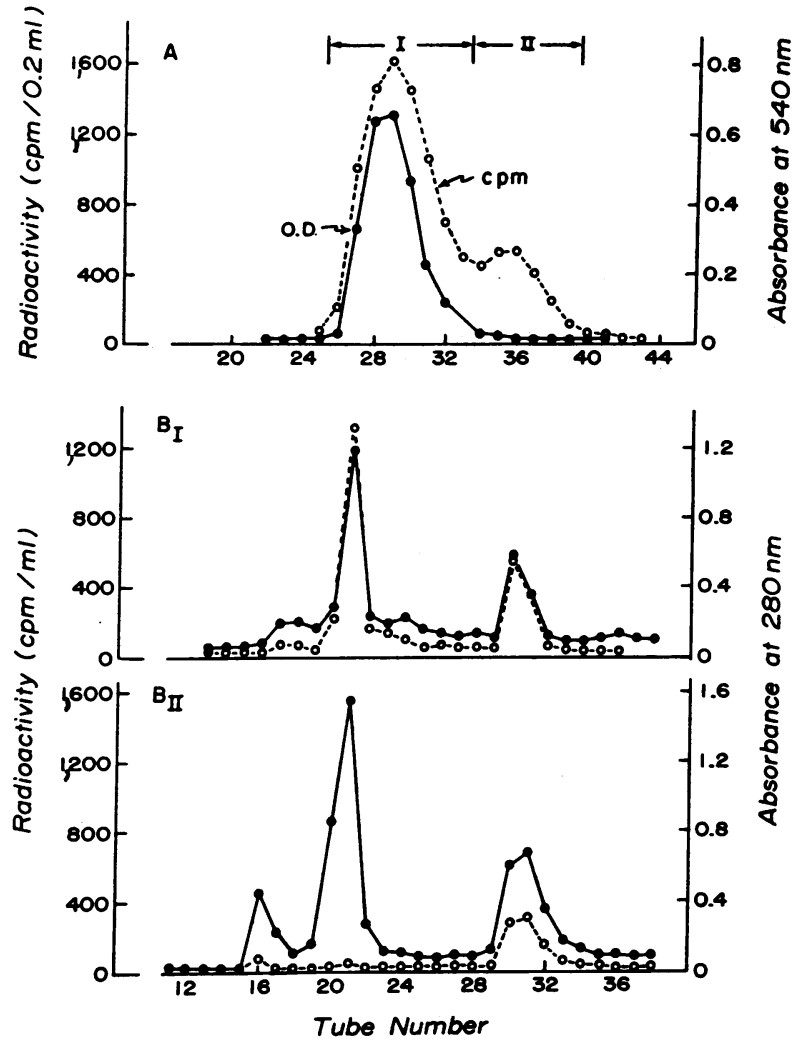

FIGURE 2 Studies of bone marrow from a patient with $\beta$-thalassemia trait (H. A.). A. Gel filtration chromatogram showing $\mathrm{Hb}$ peak (I) and peak 2 (II). $\mathrm{B}_{\mathrm{I}}$ shows the separation of globin chains from the $\mathrm{Hb}$ peak on carboxymethyl cellulose (CMC). There is radioactivity in the first peak ( $\beta$-chain) and the second peak ( $\alpha$-chain). $B_{I I}$ is the chromatogram on CMC of globin chains from peak 2 with radioactivity present only in the $\alpha$-peak.

$\beta$-thalassemia. The three peaks obtained from hemolysate from the homozygote correspond to proteins of approximate molecular weights of $64,000,32,000$, and 16,000 , presumably tetramers, dimers, and monomers of globin. One patient with sickle $\beta$-thalassemia (S. B.) had two peaks immediately after the hemoglobin peak, while the remainder of patients with sickle $\beta$-thalassemia, $\beta$-thalassemia trait, $\mathrm{Hb}$ Lepore trait, and $\boldsymbol{\alpha} \boldsymbol{\beta}$-thalassemia had only one peak. No absorbance at $540 \mathrm{~nm}$ was detected in any of the peaks except the hemoglobin peak.

Fig. 2 shows the separation of whole hemolysate by gel filtration chromatography of a sample from a patient with $\beta$-thalassemia trait, together with the globin chain separations from the two peaks recovered. The hemoglobin peak contained $\alpha$ - and $\beta$-chain radioactivity, with a $\beta / \alpha$ ratio of 1.86 . Peak 2 was composed mainly of $\alpha$-chains, with a $\beta / \alpha$ ratio of 0.09 . In order to calculate the relative amount of $\alpha$-chain radioactivity in the free $\alpha$-chain pool, a correction for $\beta$-chain radioactivity

Free $\alpha$-Globin Pool in Human Bone Marrow 


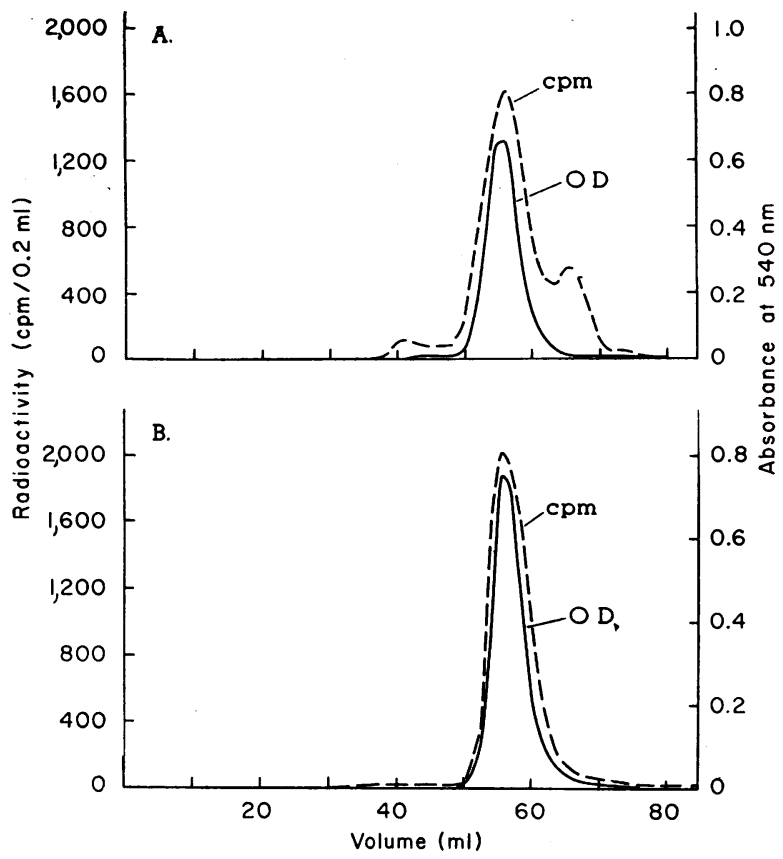

FIGURE 3 Gel filtration elution patterns from patient $H$. A. with $\beta$-thalassemia trait. A. Hemolysate applied to column immediately after preparation. B. Hemolysate stored for 4 wk at $4^{\circ} \mathrm{C}$. After storage, peak 2 is no longer present.

present in the lower molecular weight peak(s) was applied. The small amount of $\beta$-chain present in the dimer peak was assumed to be due to tetramers present from overlap with the hemoglobin peak or from $\alpha \beta$-dimers (19). The amount of radioactive $\alpha$-chain combined with the $\beta$-chain should be subtracted from the total $\alpha$-chain radioactivity in the smaller molecular weight peaks in order to determine the free $\alpha$-chain pool. Although a precise determination of this radioactivity could not be made, the most likely estimate was that the $\beta / \alpha$ ratio in these tetramers or dimers would be the same as that in the tetramers of the hemoglobin peak. The $\alpha$-chain correction was calculated on this basis and was subtracted from the total $\alpha$-chain radioactivity in the dimer and monomer peaks. For the experiments shown in Fig. 2, the $\alpha$-chain in peak 2 contained $821.0 \mathrm{cpm}$ (based on $\mathrm{cpm} / \mathrm{ml}$ ), and the $\beta$-chain contained $72.6 \mathrm{cpm}$. The $\beta / \alpha$ ratio in the hemoglobin peak was 1.86 . The $\alpha$-chain radioactivity associated with $\beta$-chain in peak 2 was calculated to be $72.6 / 1.86=39.0 \mathrm{cpm}$. The radioactive pool of free $\alpha$-chains was thus $821.0-39.0=782.0 \mathrm{cpm}$. The total $\alpha$-chain radioactivity in the hemoglobin peak and peak 2 was $1799.0 \mathrm{cpm}$. The fraction of radioactive $\alpha$-chains in the free $\alpha$-chain pool was thus $782.0 / 1799.0=$ 0.434 , or $43.4 \%$ of newly synthesized $\alpha$-chains.

Table I shows the results of similar studies on all the patients with heterozygous hemoglobin disorders and on the controls. The $\beta / \alpha$ ratios in the hemoglobin peak were either greater than or equal to the corresponding ratios in the bone marrow specimens before separation by gel filtration. The hemoglobin peak $\beta / \alpha$ ratios were increased in those patients who had relatively large free $\alpha$-chain pools. The heterozygotes had $\beta / \alpha$ ratios in peak 2 that were considerably decreased, indicating an excess of free $\alpha$-chains. The $\beta / \alpha$ ratio of total radioactivity contained in the hemoglobin peak and peak 2 was close to one in most of the patients. In particular, the ratios in the patients with $\beta$-thalassemia trait and sickle- $\beta$-thalassemia were all within the normal range. The $\beta / \alpha$ ratio in one patient with $\mathrm{Hb}$ Lepore trait (A. S.) was particularly low $(0.59)$, as was her original bone marrow ratio $(0.85)$. The mean of the original bone marrows for the patients heterozygous for $\beta$-thalassemia or $\mathrm{Hb}$ Lepore was 0.96 , and the mean for total $\beta / \alpha$ in the gel filtration peaks was 0.87 . If the particularly low values in the one patient with $\mathrm{Hb}$ Lepore trait are not included, the means are 0.98 and 0.91 . The mean radioactivity in the free $\alpha$-chain pool of the eight patients with $\beta$-thalassemia trait, sickle$\beta$-thalassemia, and $\mathrm{Hb}$ Lepore trait was $33.0 \%$ (14.0 $59.5 \%)$, while the mean of the controls was $6.2 \%$ (3.7$9.8 \%$ ). The patient with $\alpha \beta$-thalassemia had $14.1 \%$ radioactivity in his free $\alpha$-chain pool, as would be expected from the modifying influence of $\alpha$-thalassemia trait on $\alpha$-thalassemia trait.

It has been suggested (20) that a protein of high molecular weight co-chromatographing with the $\beta$-globin on urea chromatography might explain the finding of balanced synthesis in the bone marrow of $\beta$-thalassemia heterozygotes. In our gel filtration studies the amount of radioactivity associated with high molecular weight substances in the void volume was very small in comparison with the other protein peaks. It was less than $10 \%$ of the radioactivity in the hemoglobin peak in each patient in whom it was measured (11 patients), with lowest values being $0.4 \%$ of the counts in the hemoglobin peak.

Samples of bone marrow hemolysate from two patients with $\beta$-thalassemia trait, one with sickle $\beta$-thalassemia, one with $\alpha \beta$-thalassemia, and a control were kept at $4^{\circ} \mathrm{C}$ for $2-4 \mathrm{wk}$ and then applied to a Sephadex G-100 column. In each instance peak 2 was absent in the later study. Table II and Fig. 3 show the data from two experiments on a patient with $\beta$-thalassemia trait (H. A.) done $1 \mathrm{mo}$ apart, in which identical volumes of hemolysate from the same bone marrow specimen were analyzed by gel filtration chromatography followed by carboxymethyl cellulose chromatography. The ratios of $\beta / \alpha$ in the orignal marrow and in the total $\beta / \alpha$ recovered from Sephadex G-100 chromatography on two occasions were similar $(0.97,1.03$, and 0.91$)$, indicating that there was no selective loss of $\alpha$-chain during the period 
at $4^{\circ} \mathrm{C}$. The total $\alpha$-chain radioactivity initially was 12 ,$784 \mathrm{cpm}$ and the $\beta$-chain 13,216, while after $4 \mathrm{wk}$ the $\alpha$-chain had $13,475 \mathrm{cpm}$ and the $\beta$-chain $12,339 \mathrm{cpm}$. There was thus no loss of radioactivity during storage, but the $\alpha$-chain counts in the smaller molecular weight peak shifted to the tetramer peak.

Bone marrows from four children with homozygous $\beta$-thalassemia and one child heterozygous for $\beta$-thalassemia and $\mathrm{Hb}$ Lepore were also studied. Two globin peaks eluted after the hemoglobin peak in each experiment. The hemoglobin peak in each study contained radioactivity associated with $\gamma_{-}, \beta_{-}$, and $\alpha$-chains, while peaks 2 and 3 contained almost entirely $\alpha$-chain radioactivity. The pools of newly synthesized free $\alpha$-chain were 42.4 and $70.9 \%$ of the total $\alpha$-chain in the patients with thalassemia major, and $47.1 \%$ in the patient with $\beta$-thalassemia and $\mathrm{Hb}$ Lepore. The latter patient had a second bone marrow aspiration 6 wk later with a new radioactive study demonstrating a radioactive free $\alpha$-chain pool of $45.8 \%$, suggesting that the size of the pool of free $\alpha$-chains remains relatively stable in each patient.

\section{DISCUSSION}

A minor peak of radioactive protein that elutes after the hemoglobin peak on gel filtration chromatography (Sephadex G-100) of hemolysates has been previously found in rabbit and human peripheral blood and rabbit bone marrow $(6,7,21,22)$. The radioactivity was primarily associated with $\alpha$-chains, presumably as dimers or monomers. There was less radioactive $\alpha$-chain than $\beta$-chain in the tetramer peak, but the total $\alpha$-chain radioactivity in the hemolysate equaled that of $\beta$-chain. Newly formed radioactive $\alpha$-chains were presumably diluted into a small pool of nonradioactive $\alpha$-chains that was present before incubation. The newly formed radioactive $\beta$-chains combined with $\alpha$-chains from this diluted pool to form hemoglobin. The specific activity of $\alpha$-chain in the hemoglobin peak was less than that of $\beta$-chain because of the dilution of newly formed $\alpha$-chains in the nonradioactive $\alpha$-chain pool. The fraction of the newly formed $\alpha$-chains that remains in the free $\alpha$-chain is dependent on the initial size of the pool: the larger the pool, the greater the fraction of radioactive $\alpha$-chains contained in it at the end of incubation.

In this study a pool of free $\alpha$-chains has been found in the bone marrow of three control patients. The percentage of total $\alpha$-chain radioactivity contained in this pool was low (3.7-9.8\%), indicating that the size of the pre-existing pool was very small, similar in magnitude to that of human peripheral blood. This pool cannot be detected by globin synthesis studies using globin chain separation on carboxymethyl cellulose in $8 \mathrm{M}$ urea, because the method is relatively insensitive in detecting
TABLE II

Bone Marrow Globin Synthesis and Gel Filtration Data (Patient H. A.)

\begin{tabular}{ccc}
\hline & $\begin{array}{c}\text { Radio- } \\
\text { activity } \\
\text { ratio } \\
\beta / \alpha\end{array}$ \\
activity & total cpm & \\
Globin synthesis & & 0.97 \\
Immediate gel filtration & & 1 \\
Hb peak & 12,709 & \\
$\beta$ & 7,056 & \\
$\alpha$ & & 0.09 \\
Peak 2 & 507 & \\
$\beta$ & 5,728 & \\
$\alpha$ & & 1.03 \\
Hb peak + peak 2 & & \\
Repeat gel filtration* & & 0.91 \\
Hb peak & 12,339 & \\
$\beta$ & 13,475 & \\
$\alpha$ & & \\
\hline
\end{tabular}

* Specimen was stored for 4 wk at $4^{\circ} \mathrm{C}$.

small differences in amounts of pre-existing $\alpha_{-}$and $\beta_{-}$ chains.

In the patients heterozygous for $\beta$-thalassemia or $\mathrm{Hb}$ Lepore, a much larger fraction of $\alpha$-chain radioactivity was contained in the $\alpha$-chain pool (14.0-59.5\%), despite balanced production of globin chains determined by synthesis studies. In each study, newly prepared hemolysate was applied immediately to a gel filtration column to minimize exchange of globin between hemoglobin tetramers and free $\alpha$-chains $(7,23)$. This form of exchange was evident in samples kept at $4^{\circ} \mathrm{C}$ for $2-4 \mathrm{wk}$ and then restudied. The recovery of the total original radioactivity in the tetramer peak, without any residual radioactivity in the free $\alpha$-chain pool, indicates clearly that the radioactive $\alpha$-chains originally found in the pool were intact and able to combine with $\beta$-chains. Previous experiments have shown that the addition of free $\beta$-chains shifts radioactivity from the free $\alpha$-chain pool in thalassemia major to the tetramer peak $(6,7,9)$. In addition, the recovery of the total $\alpha$-chain radioactivity in the tetramer peak after a storage period indicates that the free $\alpha$-chain pool must be extremely small in comparison with the amount of globin in the tetramer peak. The distribution of $\alpha$-chain radioactivity between the tetramer and smaller peaks after equilibrium is reached should indicate the relative amounts of nonradioactive $\alpha$-chain in each peak. In the experiment shown in Fig. 3 there was no radioactive smaller peak or shoulder detectable after $2-4 \mathrm{wk}$, indicating that the free pool was very small. A shoulder containing approximately $150 \mathrm{cpm}$ or more of radioactive $\alpha$-chain would be easily detected by comparison 
with the 540-nm absorbance peak. The free $\alpha$-chain pool present in the original sample was thus less than $2 \%$ of the total globin present $[(150 / 7654) \times 100]$, or $4 \%$ of the $\alpha$-chain and possibly considerably less than this amount. A pool of this size would not be detected by the usual globin synthesis studies. Since all the initial radioactivity was recovered, there was no indication of precipitation of free $\alpha$-chains during the storage period.

One patient with $\alpha \beta$-thalassemia had $14.1 \%$ of the $\alpha$-chain radioactivity present in free $\alpha$-chains, a value intermediate between the means of controls $(6.2 \%)$ and heterozygotes $(33.0 \%)$. $\alpha$-thalassemia would be expected to modify the expression of $\beta$-thalassemia trait by an improvement in the peripheral blood globin synthesis ratio (12) and a decrease in the size of the free $\alpha$-chain pool (2).

A previous study demonstrated the presence of a large pool of free radioactive $\alpha$-chains in the bone marrow of one patient with homozygous $\beta$-thalassemia (7). The studies described here demonstrate a similar finding in four more patients and in one patient doubly heterozygous for $\beta$-thalassemia and $\mathrm{Hb}$ Lepore. The radioactivity in the $\alpha$-chain pools ranged from 42.4 to $70.9 \%$ of the total $\alpha$-chain radioactivity, a range overlapping but somewhat higher than that of the heterozygous patients. No absorbance at $540 \mathrm{~nm}$ was detected in the dimer or monomer peaks of these patients, nor absorbance at $280 \mathrm{~nm}$ in one patient, indicating that the total amount of $\alpha$-chains in these peaks was very small. There are at least three mechanisms by which the pool of free $\alpha$-chains may be reduced in homozygous $\beta$-thalassemia: compensatory synthesis of $\gamma$-chains and formation of $\mathrm{Hb} \mathrm{F}$, precipitation of $\alpha$-chains (24), and proteolysis of excess $\alpha$-chains (25). These processes may keep the size of the free $\alpha$-chain pool just slightly above that found in heterozygotes. In each of the heterozygotes in this study the level of $\mathrm{Hb} \mathrm{F}$ was low, and there were no $\alpha$-chain inclusions seen in the bone marrow (24), suggesting that the mechanisms for removing free $\alpha$-chains in thalassemia major are not prominent in these heterozygotes. Previously the presence of both dimer and monomer peaks has only been demonstrated in thalassemia major. One patient with sickle-thalassemia in this study had dimer and monomer peaks, with $31.4 \%$ free radioactive $\alpha$-chains. The reason for the appearance of one or two $\alpha$-globin peaks is not known.

Despite decreased $\beta$-chain synthesis in peripheral blood reticulocytes in heterozygous $\beta$-thalassemia, sickle- $\beta$ thalassemia, and $\mathrm{Hb}$ Lepore trait, there is balanced synthesis of $\alpha$ - and $\beta$-chains in the bone marrow of most of these patients $(14-16,26,27)$. In several patients the $\beta / \alpha$ ratio in the bone marrow was closer to unity than in the peripheral blood, but balanced synthesis was not achieved (patient A. S. in this study and references 20, 27, 28).
Patient A. S. had the highest free $\alpha$-chain pool (59.5\% of the total radioactive $\alpha$-chain) of the heterozygotes in this study. She was the most severely affected clinically of the simple heterozygotes for $\mathrm{Hb}$ Lepore or $\beta$-thalassemia in our studies (hemoglobin concentration 10.6 $\mathrm{g} / 100 \mathrm{ml}$, reticulocytes $7.7 \%$ ). One of the reported patients whose $\beta / \alpha$ bone marrow ratio was not in the normal range (sickle $\beta$-thalassemia) also had a decreased hemoglobin level, $8.0 \mathrm{~g} / 100 \mathrm{ml}(27)$. The clinical status of the other patients with low bone marrow synthesis ratios was not described $(20,28)$.

A question has been raised whether the normal or near-normal bone marrow synthesis ratios in heterozygotes are due to the combined effect of contamination of the $\beta$-chain peak with a radioactive high molecular weight protein and of extremely rapid degradation of excess $\alpha$-chain in the bone marrow (20). In our studies combining gel filtration and separation of globin chains we did not find evidence of a high molecular weight protein in the $\beta^{\boldsymbol{A}}$-peak. In addition, balanced globin synthesis in the bone marrow was found in 10 patients with sickle $\beta$-thalassemia $(16,26,27)$, where the major non- $\alpha$-chain $\left(\beta^{\mathbf{S}}\right)$ elutes at a different position from the $\beta^{\mathbf{A}}$-chain. Analysis of specific activity of tryptic peptides from the bone marrow $\beta^{\mathbf{A}}$-peak of a patient with $\mathrm{Hb}$ Lepore trait failed to reveal contamination by a nonhemoglobin protein (28). It would be extremely unlikely that approximately one-half of the newly formed $\alpha$-chains were precipitated or degraded almost immediately upon formation. The gel filtration studies did not reveal radioactive fragments of molecular weights intermediate between those of intact globin and leucine, nor were $\alpha$-chain inclusions seen on inspection of bone marrow incubated with methyl violet. Small oligopeptides would not have separated from the leucine peak in the gel filtration experiments, but larger fragments would have been detected. The $\alpha / \beta$ bone marrow ratio in a patient with $\mathrm{Hb}$ Lepore trait did not fall during several intervals in a 120 -min period, providing evidence that $\alpha$-chain was not being differentially destroyed during the incubation (28). In addition, the mean cell hemoglobins of the heterozygotes indicate that there is more $\alpha$-chain present than there would be if a major portion of the total $\alpha$-chain produced were rapidly degraded.

The data currently available from studies on a variety of heterozygotes indicate that there is compensation in the bone marrow by the nonthalassemic $\beta$-allele for the defect in the thalassemic or Lepore allele (14-16). There may also be a slight decrease in $\alpha$-chain synthesis in some patients. The synthesis of $\beta$-chain by the normal allele in persons with heterozygous $\beta$-thalassemia or $\mathrm{Hb}$ Lepore trait decays more rapidly than that of the $\alpha$-chain, resulting in decreased $\beta / \alpha$ ratios in the peripheral blood. It is likely that there is some form of regulation of 
$\beta$-chain synthesis that results in balanced globin production in the bone marrow. The nature of this regulation, whether on a transcriptional or translational level or both, is unknown. Excess $\alpha$-chains stimulate $\beta$-chain synthesis and depress $\alpha$-chain synthesis in hemolysates of rabbit peripheral blood (29). The regulation of balanced globin synthesis by a small pool of free $\alpha$-chains in normal bone marrow and the stimulation of compensatory synthesis of $\beta$-chains by a larger pool of $\alpha$-chains in bone marrow of heterozygotes is a hypothesis deserving of further investigation.

\section{ACKNOWLEDGMENTS}

We wish to thank Veronica Watson and Margaret Martin for expert technical assistance.

This work was supported by U. S. Public Health Service Grant AM 16691 and by grants from the Cooley's Anemia Foundation and the Commonwealth of Pennsylvania.

\section{REFERENCES}

1. Schwartz, E., and F. M. Gill. 1971. Evidence for the existence of a small pool of free $\alpha$-chains in heterozygous beta thalassemia bone marrow. Clin. Res. 19: 430.

2. Gill, F. M., and E. Schwartz. 1972. Free alpha-chain pool in the bone marrow. Pediatr. Res. 6: 370 .

3. Weatherall, D. J., J. B. Clegg, and M. A. Naughton. 1965. Globin synthesis in thalassemia: an in vitro study. Nature (Lond.). 208: 1061.

4. Bank, A., and P. A. Marks. 1966. Excess alpha chain synthesis relative to beta chain synthesis in thalassemia major and minor. Nature (Lond.). 212: 1198.

5. Kan, Y. W., E. Schwartz, and D. G. Nathan. 1968. Globin chain synthesis in the alpha thalassemia syndromes. J. Clin. Invest. 47: 2515.

6. Huehns, E. R., and C. B. Modell. 1967. Symposium on thalassaemia. Haemoglobin synthesis in thalassaemia. Trans. R. Soc. Trop. Med. Hyg. 61: 157.

7. Modell, C. B., A. Latter, J. H. Steadman, and E. R. Huehns. 1969. Haemoglobin synthesis in $\beta$-thalassaemia. Br. J. Haematol. 17 : 485.

8. Conconi, F., A. Bargellesi, S. Pontremoli, V. Vigi, S. Volpato, and D. Gaburro. 1968. Absence of $\beta$-globin synthesis and excess of $\alpha$-globin synthesis in homozygous $\beta$-thalassemia subjects from the Ferrara region. Nature (Lond.). $217: 259$.

9. Bank, A., and J. V. O'Donnell. 1968. Hemoglobin synthesis in $\beta$-thalassemia: the properties of the free $\alpha$ chains. J. Clin. Invest. $47: 860$.

10. Weissman, S. M., I. Jeffries, and M. Karon. 1967. The synthesis of alpha, beta, and delta peptide chains by reticulocytes from subjects with thalassemia or hemoglobin Lepore. J. Lab. Clin. Med. 69: 183.

11. Bank, A., A. S. Braverman, J. V. O'Donnell, and P. A. Marks. 1968. Absolute rates of globin chain synthesis in thalassemia. Blood J. Hematol. 31: 226.
12. Kan, Y. W., and D. G. Nathan. 1970. Mild thalassemia : the result of interactions of alpha and beta thalassemia genes. J. Clin. Invest. 49: 635.

13. Weatherall, D. J., J. B. Clegg, S. Na-Nakorn, and P. Wasi. 1969. The pattern of disordered hemoglobin synthesis in homozygous and heterozygous $\beta$-thalassemia. Br. J. Haematol. 16: 251.

14. Schwartz, E. 1970. Heterozygous beta thalassemia: balanced globin synthesis in bone marrow cells. Science (Wash. D. C.). 167: 1513.

15. Gill, F., J. Atwater, and E. Schwartz. 1972. Hemoglobin Lepore trait: globin synthesis in bone marrow and peripheral blood. Science (Wash. D. C.). 178:623.

16. Gill, F. M., and E. Schwartz. 1973. Synthesis of globin chains in sickle $\beta$-thalassemia. J. Clin. Invest. 52: 709.

17. Clegg, J. B., M. A. Naughton, and D. J. Weatherall. 1965. An improved method for the characterization of human hemoglobin mutants: identification of $\alpha_{2} \beta_{2}{ }^{86 \mathrm{Glu}}$ hemoglobin N (Baltimore). Nature (Lond.). 207: 945.

18. Kan, Y. W., E. Schwartz, and D. G. Nathan. 1968. Unbalanced globin chain synthesis in the alpha-thalassemia heterozygotes. Ann. N. Y. Acad. Sci. 165: 288.

19. Antonini, E., and M. Brunori. 1971. Hemoglobin and Myoglobin in their Reactions with Ligands. American Elsevier Publishing Co., Inc., New York. 110.

20. Clegg, J. B., and D. J. Weatherall. 1972. Haemoglobin synthesis during erythroid maturation in $\beta$-thalassemia. Nat. New Biol. 240: 190.

21. Tavill, A. S., A. I. Grayzel, I. M. London, M. K. Williams, and G. A. Vanderhoff. 1968. The role of heme in the synthesis and assembly of hemoglobin J. Biol. Chem. 243: 4987.

22. Tavill, A. S., G. A. Vanderhoff, and I. M. London. 1972. The control of hemoglobin synthesis. A comparison of the role of heme in rabbit bone marrow and reticulocytes. J. Biol. Chem. 247: 326.

23. Bunn, H. F., and J. H. Jand1. 1968. Exchange of heme among hemoglobins and between hemoglobin and albumin. J. Biol. Chem. 243 : 465.

24. Fessas, P. 1963. Inclusions of hemoglobin in erythroblasts and erythrocytes of thalassemia. Blood J. Hematol. $21: 21$.

25. Bank, A., and J. V. O'Donnell. 1969. Intracellular loss of free $\alpha$-chains in $\beta$-thalassemia. Nature (Lond.). 222: 295 .

26. Kan, Y. W., D. G. Nathan, and H. F. Lodish. 1972. Equal synthesis of $\alpha$ - and $\beta$-globin chains in erythroid precursors in heterozygous $\beta$-thalassemia. J. Clin. Invest. 51 : 1906.

27. Bank, A., L. W. Dow, M. J. Farace, J. V. O'Donnell, S. Ford, and C. Natta. 1973. Changes in globin synthesis with erythroid cell maturation in sickle thalassemia. Blood J. Hematol. 41 : 353.

28. White, J. M., A. Lang, and H. Lehmann. 1972. Compensation of $\beta$-chain synthesis by the single $\beta$-chain gene in Hb Lepore trait. Nat. New Biol. 240: 271.

29. Blum, N., N. Maleknia, and G. Schapira. 1970. Régulation de la synthèse de l'hémoglobine par ses subunites. Bull. Soc. Chim. Biol. 52: 1279. 